\title{
Cardio-Thoracic Ratio Measurement Using Non-linear Least Square Approximation and Local Minimum
}

\author{
Wasin Poncheewin ${ }^{1 *}$, Monravee Tumkosit ${ }^{2}$, Rajalida Lipikorn ${ }^{1}$ \\ ${ }^{1}$ Machine Intelligence and Multimedia Information Technology Laboratory, Department of Mathematics and \\ Computer Science, Faculty of Science, Chulalongkorn University, Bangkok, Thailand. \\ ${ }^{2}$ Department of Radiology, Faculty of Medicine, Chulalongkorn University, Bangkok, Thailand. \\ * Corresponding author. Tel.: +66835435313; email: wasin_poncheewin@hotmail.com \\ Manuscript submitted January 10, 2015; accepted May 8, 2015. \\ doi: $10.17706 /$ jcp.10.5.321-328
}

\begin{abstract}
This paper presents a method to automatically measure cardio-thoracic ratio (CTR) from a chest radiographic images using non-linear least square approximation and local minimum. The proposed method consists of initial boundary point identification, cardiac diameter measurement, thoracic diameter measurement and cardio-thoracic ratio measurement. First, the initial boundary points used to approximate the region of thoracic cavity are identified using general human anatomy features. Then the non-linear least square approximation and local minimum are used to detect the heart boundary. Finally, the thoracic cage boundary is detected and the cardio-thoracic ratio can be measured. The proposed method is tested on a set of 255 chest radiographs. The experimental results are evaluated using correlation test between two sets of numerical measurement which are measured by our proposed method and by the radiologists. The evaluation reveals that the correlation result on CTR is about $78 \%$.
\end{abstract}

Key words: Cardio-thoracic ratio, chest radiograph, computer-aided diagnosis.

\section{Introduction}

Radiography is one of the most widely used medical procedures performed to diagnose the insight of human body. Chest radiograph is a useful tool for diagnosing the abnormality that occurs in chest cavity and also is a source to obtain the size of the heart which is one of the most important factors used to indicate many heart diseases including enlarged heart or cardiomegaly [1]. In the past, cardio-thoracic ratio (CTR) was manually measured by radiologists. At the present, computer-aided diagnosis (CAD) system has become much more popular. This technology has improved the way to diagnose many diseases by giving many advantages such as accuracy, time consumption reduction and automation [2] but a fully automated CTR measurement has not yet been able to gain a complete reliability from radiologists.

Various techniques of automated detection of cardiomegaly have been proposed. The study by M. Ilovar and L. Šajn uses edge detection to locate the position of the heart and determine the border of the thoracic cage by drawing a horizontal line toward the end of an image from the points on both sides of the heart boundary which have been calculated as the widest part and finding the brightest intensity [3]. This method of measuring the CTR is not precise according to the actual definition by Danzer, C.S. [4]. Ishida and his colleagues had developed an algorithm that uses histogram analysis and edge detection technique with feature analysis to diagnose the cardiomegaly but it is not yet fully automated [5]. Another novel method was developed by Sezaki Nobuhiko and Ukena Kohji which can compute the CTR within one second but it 
must be installed on their special instrument [6]. Beside all of the technical work, there are also a survey and a comparative study of other proposed methods that provide useful information [7], [8].

The aim of this proposed method is to develop a fully automated CTR measurement technique without the use of training data set which can improve the accuracy and efficiency. The paper is organized as follows: Section 2 presents related work and theoretical background, Section 3 proposes a new CTR measurement, Section 4 provides experimental results, and Section 5 gives some discussion and conclusions.

\section{Proposed Methodology}

In order to measure CTR, the radiographic images in DICOM format are converted to gray scale images. The input images are then passed through the proposed method which consists of four main processes: initial boundary point identification, cardiac diameter measurement, thoracic diameter measurement, and CTR measurement.

\subsection{Initial Boundary Point Identification}

In the first process, histogram equalization and median filter are used to enhance a gray scale image. Then, the average intensity is used as a threshold value to convert a gray scale image to a binary image. Next, the initial boundary points which are used to define the region of lung are identified by the following process.

1) Identify the initial center point (IC) of the body by setting the x-coordinate to equal to the middle column of the white region on the last row of a binary image of each patient which is assumed to represent the patient's body and setting the y-coordinate to be half of the height of an image. The IC point is shown as '*' in Fig. 1.

2) Identify the initial left and right boundary points (LR) by using the initial center point obtained from previous step. There are altogether six boundary points which represent the left and the right boundaries. Four pixels represent the left and the right boundaries of each lung, and two pixels represent the left and the right boundaries of the body. These six initial boundary points are identified by finding the pixels along the same row as the initial point whose gradients of intensity are not equal to 0 . The LR points are shown as ' + ' in Fig. 1.

3) Identify the initial upper and lower boundary points (UL) of the lung by finding the lower and upper $y$-coordinates of the midpoint between the left and the right boundary points of the lung whose gradients are not equal to 0 . The UL points are shown as ' $x$ ' in Fig. 1.

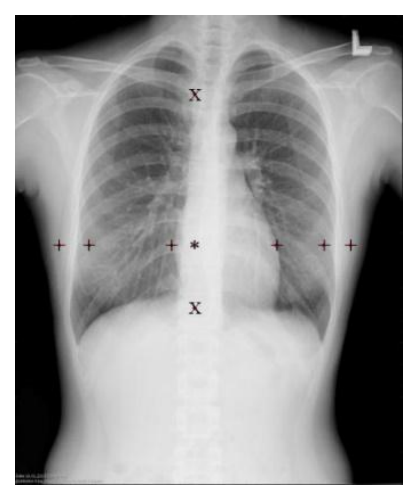

Fig. 1. Initial boundary points.

\subsection{Cardiac Diameter Measurement}

Since the cardio-thoracic ratio is computed by dividing the cardiac diameter (CD) by the thoracic 
diameter (TD), the next process is to measure cardiac diameter. Because the heart is non-rigid and asymmetry, the proposed cardiac diameter measurement divides the heart into two sides: the left and the right. It then finds the widest part of the heart on the left and the widest part of the heart on the right separately. The summation of these two values becomes the cardiac diameter. In order to find the widest parts on both sides of the heart, the proposed CD measurement computes the maximum distance from the center of the body which is a vertical line that passes through the IC point to the left side of the heart and the maximum distance from the center of the body to the right side of the heart as follows:

\subsubsection{Measurement on the left side of the heart}

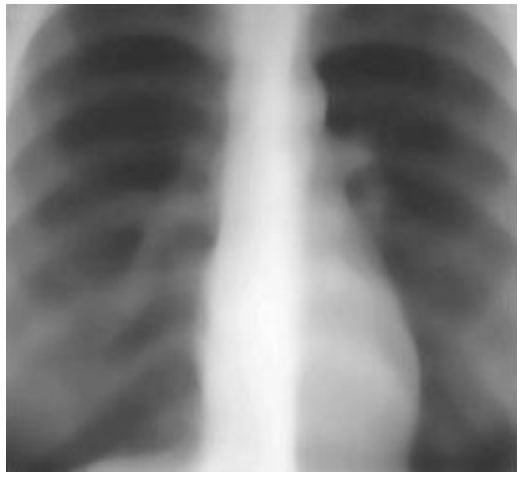

(a) The thoracic cage

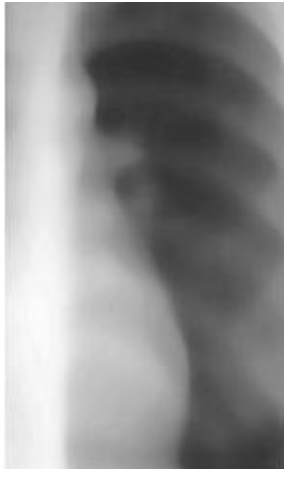

(b) The left thoracic cage

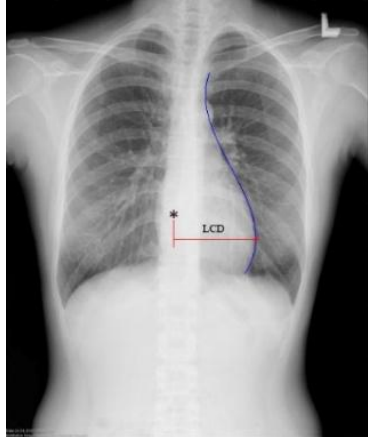

(c) The left boundary of the heart and the widest part

Fig. 2. The thoracic cage, the left thoracic cage and the left boundary of the heart and the widest part.

1) Extract the region of the whole thoracic cage from an image by using the initial boundary points (LR and UL points) obtained from the first process. The extracted region is shown in Fig. 2(a).

2) Extract the region of the left thoracic cage by segmenting the left side of the thoracic cage from the previous step. The extracted region is shown in Fig. 2(b).

3) Compute the average intensity of the whole thoracic cage and the average intensity of the left thoracic cage. Set the threshold value to equal to the minimum average intensity from these two values.

4) Convert an image of the left thoracic cage to a binary image using the threshold value obtained from step 3).

5) Detect the boundary on the left side of the heart by scanning along the vertical line that passes through the IC point to find the first pixel whose gradient is not equal to 0 .

6) Detect the left boundary of the heart by using Density-based spatial clustering of applications with noise (DBSCAN) [9] to remove noise from the boundary.

7) Connect the left boundary of the heart by using non-linear least square approximation as shown in eqn. (1), (2) and (3).

$$
E=\sum_{i=1}^{m}\left[y_{i}-P_{n}\left(x_{i}\right)\right]^{2}
$$

where $E$ represents the sum of error of $m$ data which consists of $x_{i}, y_{i}$ when $i=1,2, \ldots, m$.

$$
\frac{\partial E}{\partial a_{j}}=0
$$

In order to minimize the sum of the least square, the gradient is set to zero where $a_{j}$ is the coefficients of a polynomial $P(x)$ of degree $n, j=1,2, \ldots, n$, and the results will be used in eqn.(3).

$$
P_{n}(x)=a_{0}+a_{1} x+\cdots+a_{n-1} x^{n-1}+a_{n} x^{n}
$$

8) Find the widest part on the left side of the heart by finding the maximum distance along the vertical line 
from the center of the body to the left boundary of the heart. The left boundary and its widest part, LCD, are shown in Fig. 2(c).

\subsubsection{Measurement on the right side of the heart}

1) Extract the region of the right thoracic cage by using the initial boundary points (LR and UL points) obtained from the first process. The extract region is shown in Fig. 3.

2) Quantize an 8-bit gray scale image of the right thoracic cage to a 4-bit gray scale image and plot a histogram.

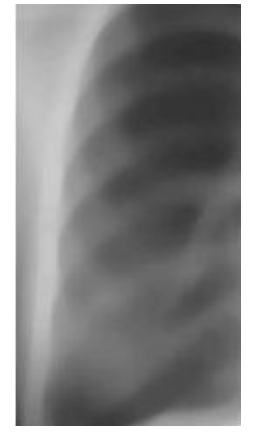

(a) The right thoracic cage

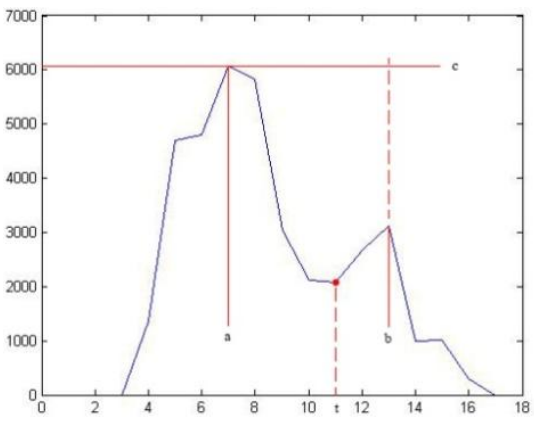

(b) Histogram of the right thoracic cage

Fig. 3. The right thoracic cage and its quantized histogram.

3) Find the valley in the histogram whose area is the maximum. The area can be computed by

$$
\text { valley }=\int_{a}^{b}(c-f(x)) d x
$$

where $c$ represents the highest point of the valley and $[a, b]$ represent the range of intensity of the valley. Then the threshold value, $t$, is set to equal to the intensity of the local minimum of the valley as shown in Fig.4.

1) Convert an image of the right thoracic cage to a binary image using the threshold value obtained from step 3).

2) Detect the right boundary of the heart by using DBSCAN to remove noise from the boundary.

3) Connect the right boundary of the heart by using the same non-linear least square approximation.

4) Find the widest part on the right side of the heart by finding the maximum distance along the vertical line from the center of the body to the right boundary of the heart. The right boundary and its widest part, RCD, are shown in Fig. 4.

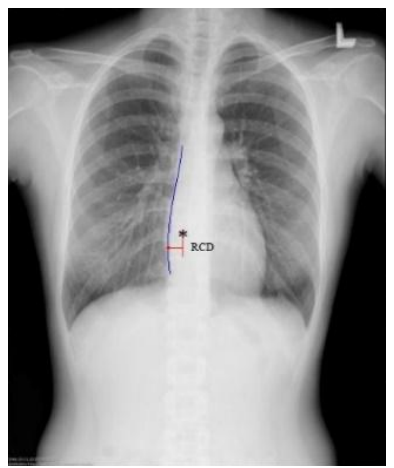

Fig. 4. The right boundary of the heart and the widest part.

\subsubsection{Thoracic diameter measurement}

In this process, the diameter of the thoracic cage is computed by first finding the widest part of the thoracic cage and then measuring the distance from the left side to the right side of the thoracic cage. The 
boundary of the thoracic cage is detected by using the average intensity to convert an image to a binary image. Then the diameter is measured by calculating the distance from the left to the right boundary of the thoracic cage along each row. The maximum diameter, TD, is shown in Fig. 5(a). Then, the CTR can be calculated by

$$
C T R=\frac{C D}{T D}
$$

where $C D=L C D+R C D$ as shown in Fig. 5(b).

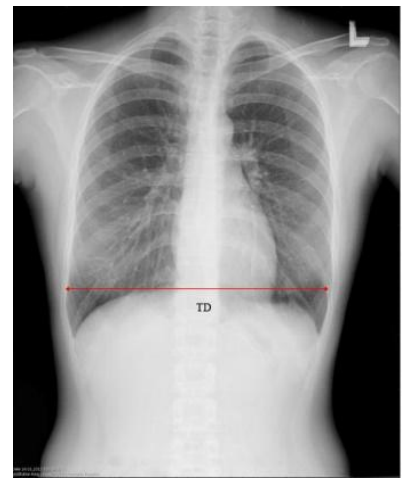

(a) The widest thoracic diameter.

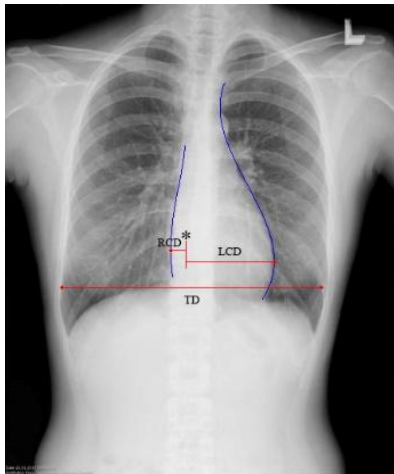

(b) The measurement of CTR.

Fig. 5. The widest thoracic diameter and the measurement of CTR.

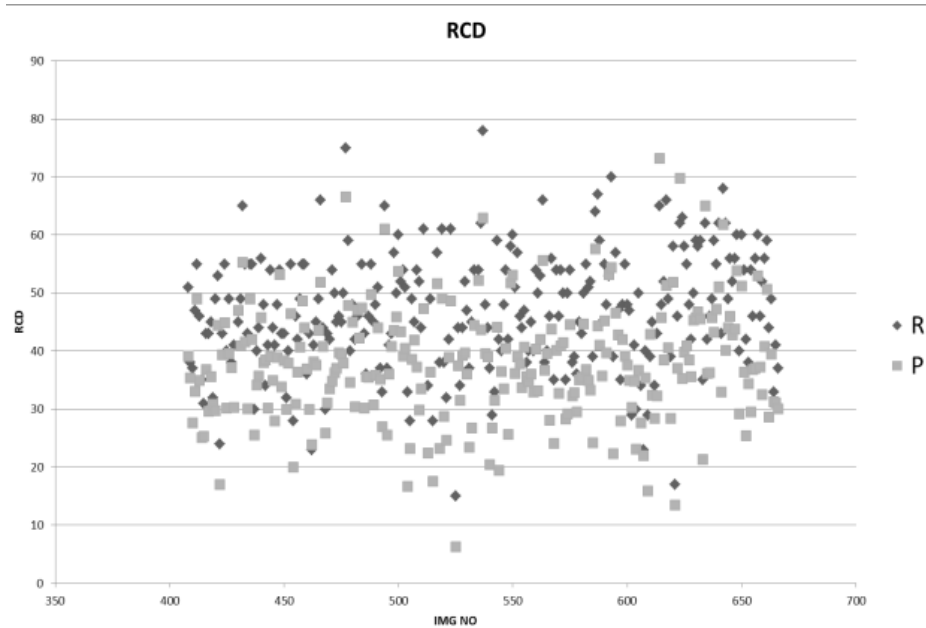

(a) RCD comparison graph

LCD

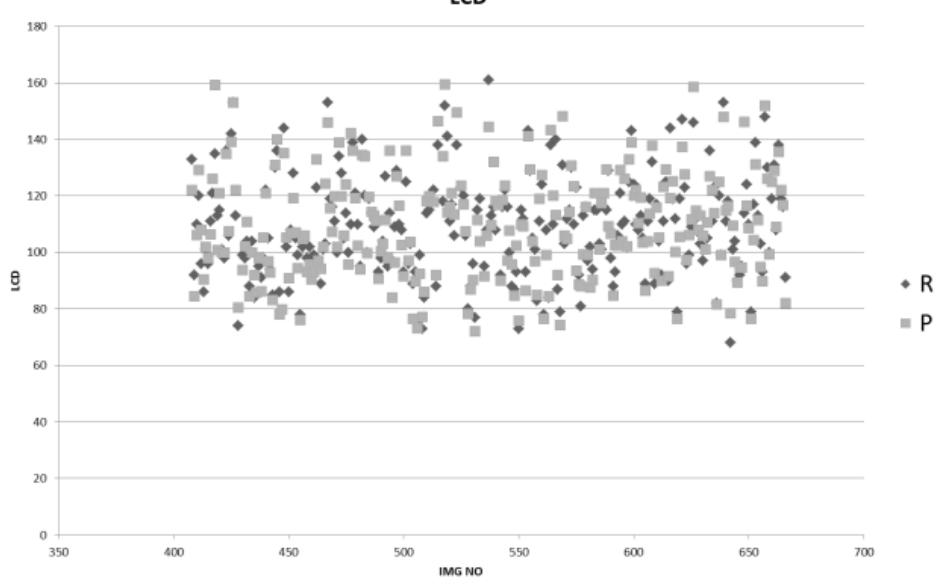

(b) LCD comparison graph

Fig. 6. RCD and LCD comparison results. 


\section{Experimental Results}

The proposed method is tested on a set of 255 chest radiographs which were taken in posterior-anterior position. All images are collected from King Chulalongkorn Memorial Hospital, Bangkok, Thailand. The format of the original images is DICOM format with a resolution of $2688 \times 2208$ pixels.

To validate efficiency of our work, a correlation test using Pearson's linear correlation coefficient is performed between two sets of numerical measurement results. The first set consists of RCD, LCD, CD, TD and CTR which were measured by our proposed technique and the second set which contains the same type of information but were measured by the radiologist. A comparison of two sets of numerical measurement results represented in Fig. 6, Fig. 7 and Fig. 8 which R is a set of the measurement result measured by the radiologist and $\mathrm{P}$ is a set of the measurement result measured by the proposed method. Some comparison results of the radiograph are displayed in Fig. 9 and the correlation results are demonstrated in Table 1. It is indicated in Fig. 9 that the numerical results between two datasets are quite similar.

Table 1. Correlation Test Results

\begin{tabular}{lllll}
\hline \hline RCD & LCD & CD & TD & CTR \\
\hline 0.7808 & 0.8957 & 0.8756 & 0.9295 & 0.7849 \\
\hline
\end{tabular}

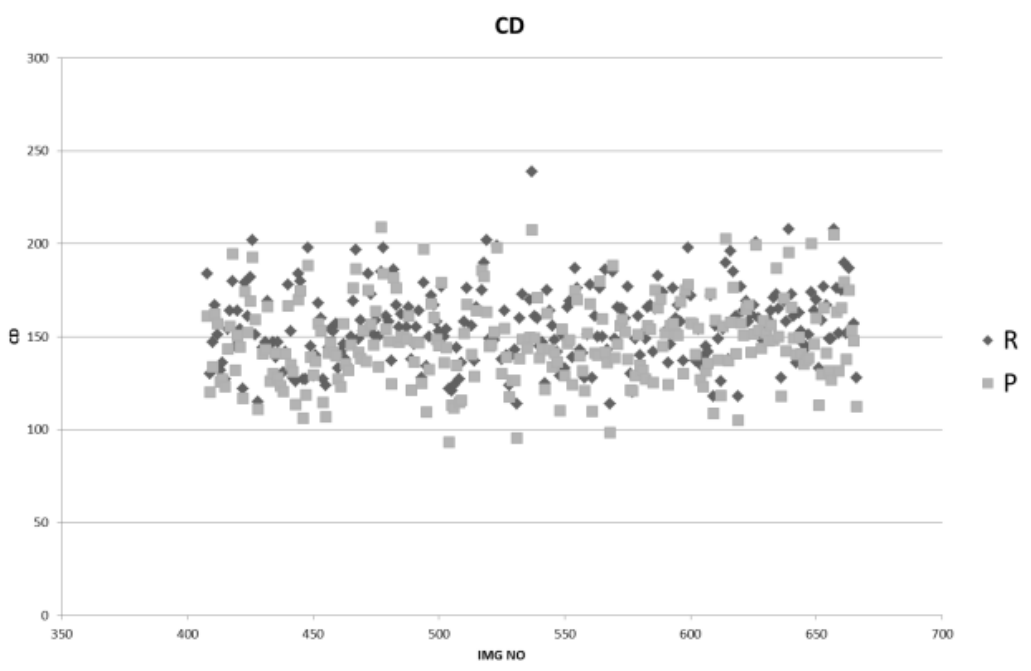

(a) CD comparison graph

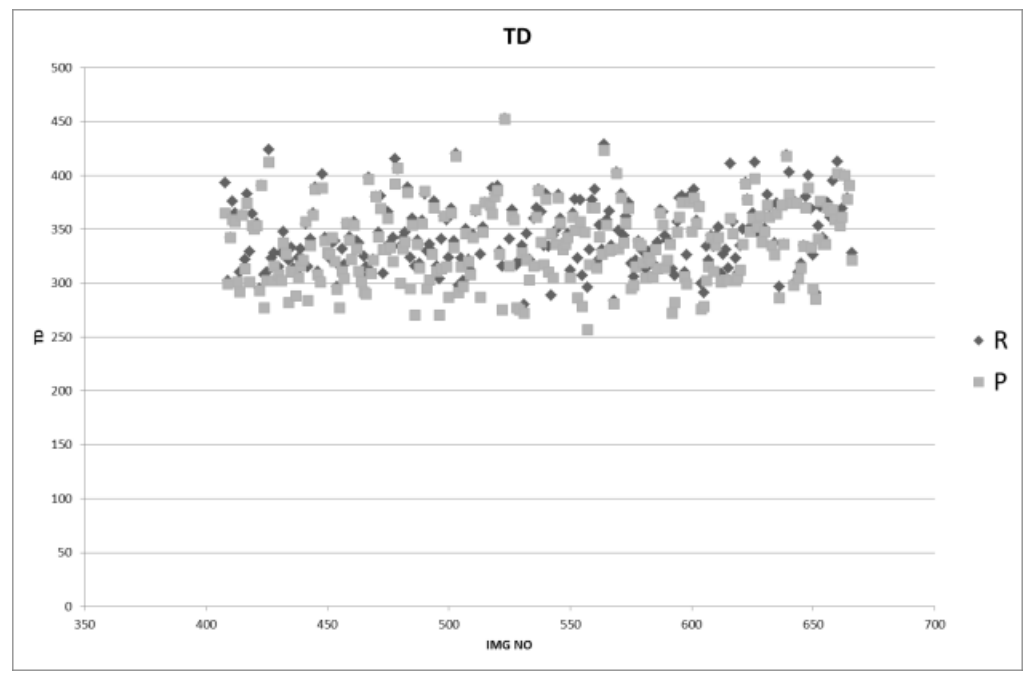

(b) TD comparison graph

Fig. 7. CD and TD comparison results. 


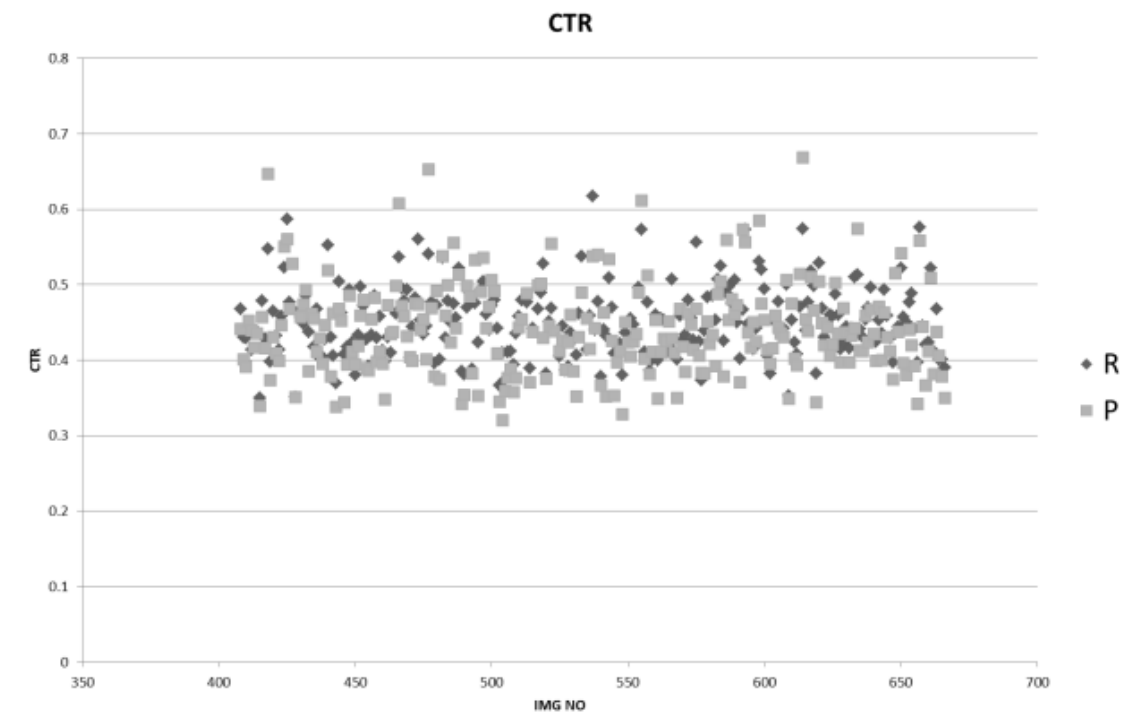

Fig. 8. CTR comparison graph.
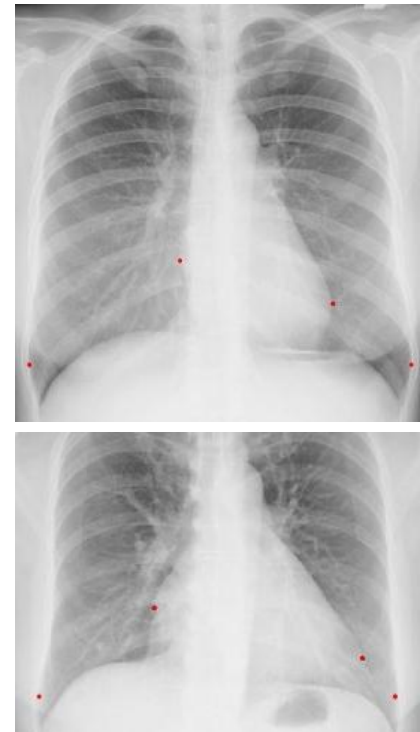

(a) The points to measure CTR selected by the radiologist.
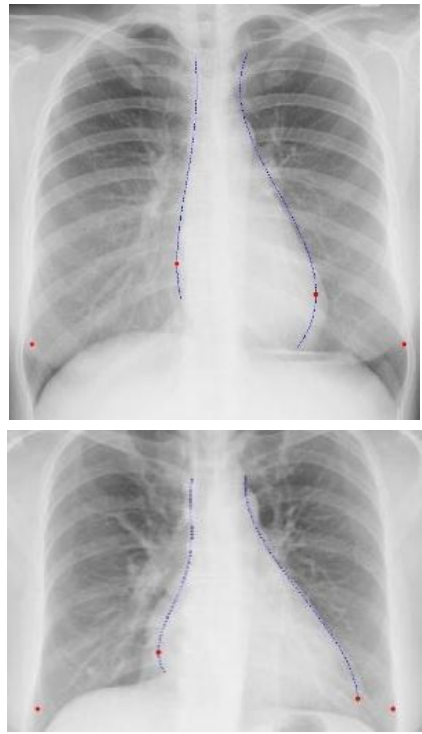

(b) The points to measure CTR selected by the proposed method.

Fig. 9. The comparison results.

\section{Conclusion}

This paper presents a new method to automatically measure the CTR from an input image of a Posterior-Anterior chest radiograph by using non-linear least square approximation and local minimum. In Table 1, the results of CTR measurement show quite a promising trend between two numerical datasets. However, these results can be improved because some of the chest radiographs might contained pericardial fat pad or vascular shadow whose intensities are similar to the heart which cause the measurement of RCD and LCD to be discrepancy.

\section{Acknowledgment}

This work is supported by Centre of Excellence in Mathematics, Thailand. We would also thank to PetcharleeyaSuwanpradit for providing medical images. 


\section{References}

[1] Longo, D., Fauci, A., Kasper, D., Hauser, S., Jameson, J., \& Loscalzo, J. (2011). Harrison's Principles of Internal Medicine (18th ed.). McGraw Hill Professional.

[2] Doi, K., MacMahon, H., Katsuragawa, S., Nishikawa, R. M., \& Jiang, Y. (1999). Computer-aided diagnosis in radiology: Potential and pitfalls. European Journal of Radiology, 31(2), 97-109.

[3] Ilovar, M., \& Sajn, L. (May 23-27, 2011). Analysis of radiograph and detection of cardiomegaly. 2011 Proceedings of the 34th International Convention (pp. 859, 863).

[4] Danzer, C. S. (1919). The cardiothoracic ratio: An index of cardiac enlargement. The American Journal of the Medical Sciences, 157(4), 513-554.

[5] Ishida, T., Katsuragawa, S., Chida, K., MacMahon, H., \& Doi, K. (May 2005). Computer-aided diagnosis for detection of cardiomegaly in digital chest radiographs, Proceedings of SPIE 5747 (p. 914).

[6] Sezaki, N., \& Ukena, K. (July 1973). Automatic Computation of the cardiothoracic ratio with application to mass screening. IEEE Transactions on Biomedical Engineering, 20(4), 248, 253.

[7] Van Ginneken, B., Viergever, M. A., et al. (Dec. 2001). Computer-aided diagnosis in chest radiography: A survey. IEEE Transactions on Medical Imaging, 20(12), 1228, 1241.

[8] Van Ginneken, B., Stegmann, M. B., \& Loog, M. (February 2006). Segmentation of anatomical structures in chest radiographs using supervised methods: A comparative study on a public database. Medical Image Analysis, 10(1), 19-40.

[9] Ester, M., Kriegel, H. P., Sander, J., \& Xu, X. (1996). A density-based algorithm for discovering clusters in large spatial databases with noise. Proceedings of the Second International Conference on Knowledge Discovery and Data Mining(KDD-96) (pp. 226-231).

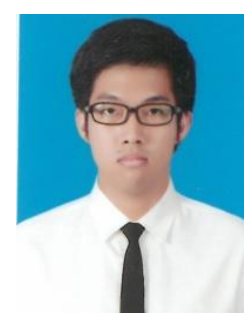

Wasin Poncheewin was born in Bangkok on June 2, 1990. He is a master's degree student majoring in computer science and information technology at the Department of Mathematics and Computer Science, Faculty of Science, Chulalongkorn University. He also graduated in a bachelor's degree with majoring in computer science at the same place in 2011.

He has an internship at Imaging Technologies Laboratory (IMG), National Electronics and Computer Technology Center (NECTEC) from March 14, 2011 to May 12, 2011.

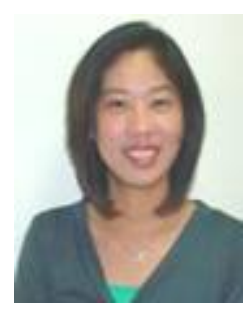

Monravee Tumkosit got the bachelor degree from the Faculty of Medicine (Honors), Chulalongkorn University with specialty in radiology and diagnostic imaging. Also, she got the diploma of The Thai Board of Diagnostic Radiology in 2005. She got the fellowships in cardiovascular imaging from Wake Forest University School of Medicine, North Carolina, USA during 2006-2007.

Her special clinical interests are general radiology and cardiovascular imaging.

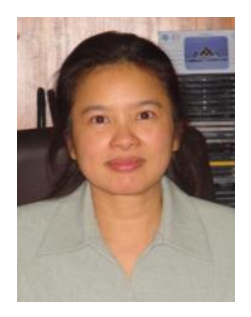

Rajalida Lipikorn is an assistant professor and a Ph.D. She got the bachelor degree in mathematics from California State University, Northridge, USA, the master degree in computer science from California State University, Northridge, USA, and the PhD degree in engineering from Tokyo University of Agriculture \& Technology, Japan.

Her research areas are in computer graphics, medical image processing, 2D and 3D object recognition. 